Faster PCR

\title{
Go with the microflow
}

\section{The demand for faster, cheaper and more reliable assay systems is driving a new technical revolution in the form of microfluidics — the flow of tiny droplets through hair-thin tubing — with applications everywhere from industry to doctors' offices. J ulie Clayton reports.}

Going small is becoming increasingly big business in the life sciences, as the desire for quicker results, higher throughput and lower costs is creating a booming market for microfluidics technology — which involves extremely small volumes of liquid. Originally a scientific curiosity of physicists and chemists, microfluidics now appears ready to transform traditional assay systems in academia and biotech as well as in big pharma and hospitals, with devices labelled as 'pinhead petri dishes' and 'Labon-a-chip'.

At its most fanciful, microfluidics could revolutionize the way in which doctors diagnose disease and monitor treatment — with portable devices for rapid automated analysis of body fluids or tissue samples, going from sample preparation to data readout in minutes or even seconds. Meanwhile, laboratory scientists are beginning to find themselves as the giant operators of a Lilliputian world in which tens or hundreds of experiments take place in parallel on 'microchips' no bigger than a credit card, reducing sample size and consumption of solvents and reagents ${ }^{1-3}$.

According to a recent market analysis ${ }^{4}$, microfluidics applications in the life sciences have a global market of around 500 million euros, which could rise to around 1.4 billion euros by 2008 , particularly if sales in drug discovery, medical diagnostics and therapeutic devices live up to their promise.

\section{Bypassing mosquitoes}

Evolution may have gotten there first with the proboscis of a mosquito for drawing up blood, but microfluidics engineers are creating their own narrow-gage devices through which tiny volumes of fluid can be propelled by laminar flow, electrophoresis or centrifugal force (see Box 1). "Nature's had

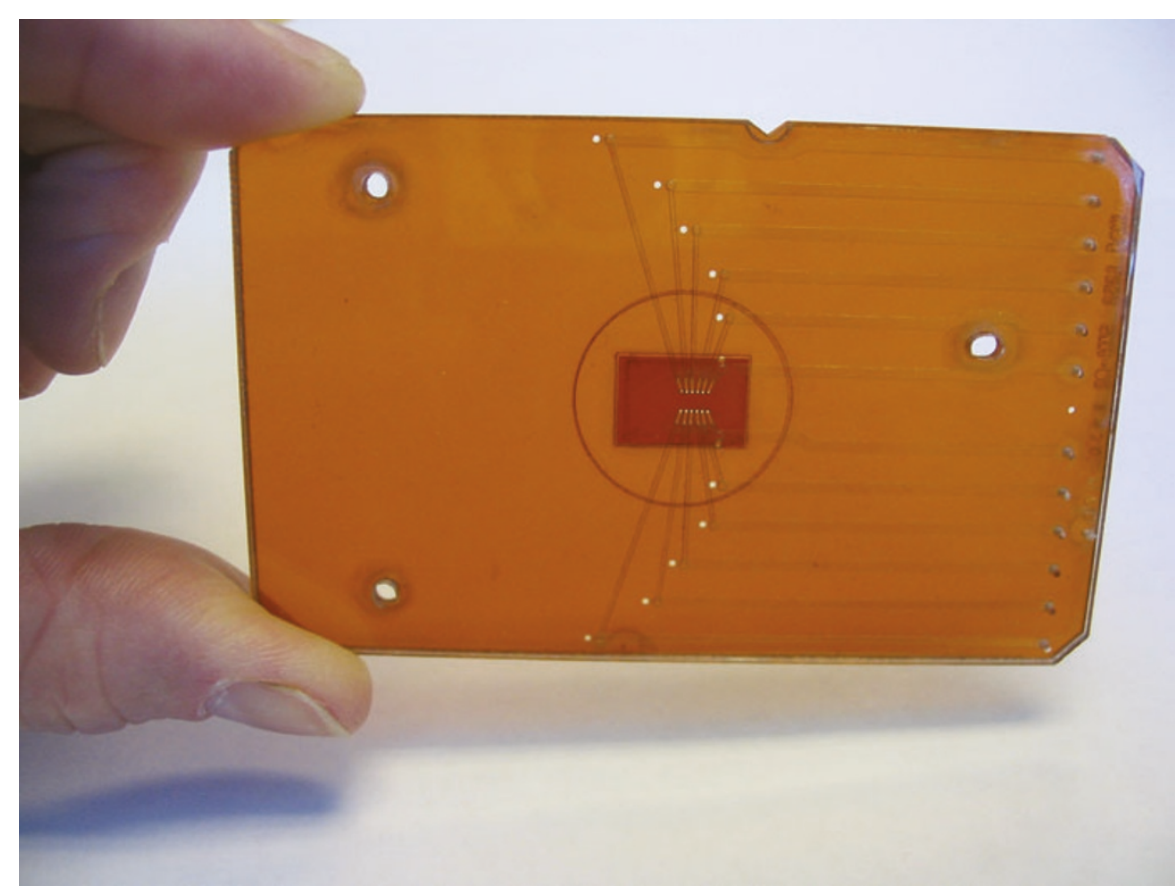

Active lab card: point-of-care diagnostic device integrating microarray in microfluidic circuitry. (Courtesy of Micronics Inc.)

millions of years to move liquids through small capillaries," says Tony Guiseppi, scientific director of ABTECH Scientific.

As he puts it, "Microfluidics technology gives us the ability to work with relatively small amounts of samples." This capability, of course, has applications in a variety of areas-from disease diagnosis to drug discovery, and the plethora of laboratory techniques for which smaller means better, faster or more convenient.

\section{Diagnostics}

Industry pundits predict that clinical diagnostics will be one of the biggest target areas for microfluidics applications, in which miniaturized bioassays can be performed more quickly and at lower cost than with conventional methods. This is driving the development of portable devices for use at patients' bedsides or in doctors' offices, thus skipping the need for more time-consuming tests in specialized laboratories. "There are few products on the market now, but it seems to be a promising field for the future-many companies are interested in this," comments Stephan Messner of Liquid Handling Competence Centre (LICOM) — an EU-funded consortium that provides microfluidics R\&D services for the life sciences.

Companies developing microfluidics 'point-of-care' tools and products include HandyLab and Micronics. Micronics offers a laminar flow-based microfluidics Lab Card prototyping service with Active, 
integrating various types of clinical chemistries, immunoassays and nucleic acid assays for a range of applications. This already forms the basis for an ABO blood typing card, licensed to Pall Corporation, which achieves a result in just ten seconds from a finger-prick drop of blood. "All reagents are on the card. You only have to introduce the sample," says Karen Hedine on of Micronics.

Micronics also plans to produce its own brand of devices for infectious disease and cancer diagnosis, involving either immunoassay or rapid (7-min) PCR, and has developed a portable Microcytometer for cell counting and cell sorting applications in healthcare or for sampling of water, soil and air for microbes.

\section{Faster PCR}

The widespread use of PCR in DNA and RNA analysis makes this an especially 음 attractive target for microfluidics techno은 logy. Companies with PCR-on-a-card techज nology include Roche, Caliper Life Sciences 을 and Cepheid. Cepheid's GeneXpert and

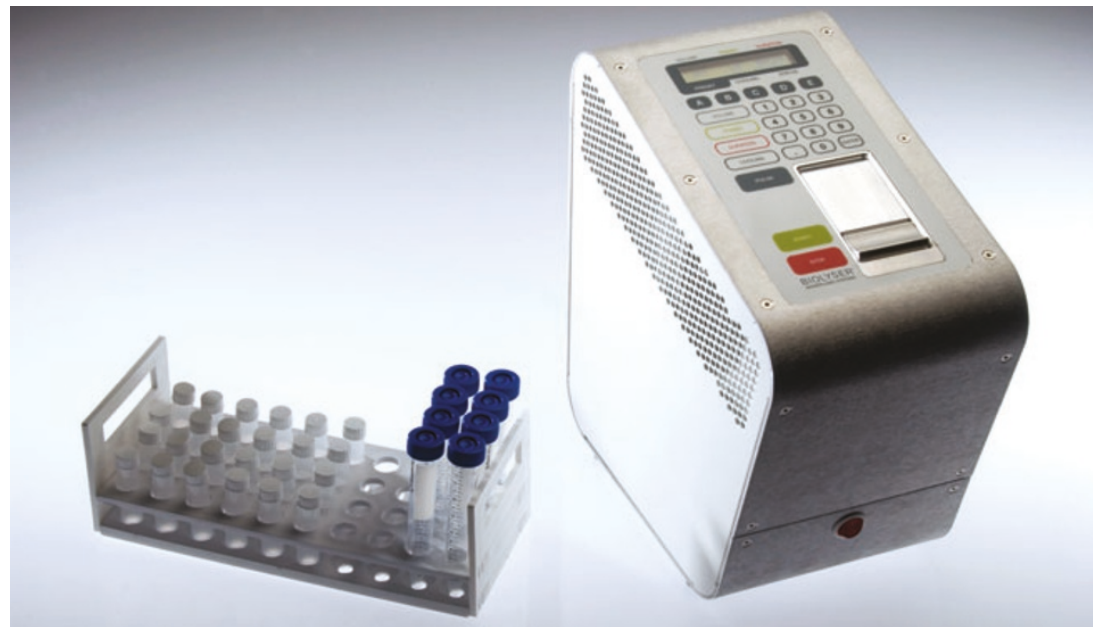

MFSI Biolyser with its programmable user interface. Multiple times and power levels, as well as active cooling, can be stored as addressable protocols on the system. Also shown are the low-cost, disposable sample vials. Bacterial spore lysis in 100-microliter volumes occurs within 20 seconds. (Courtesy of MicroFluidic Systems.)

SmartCycler extracts and tests DNA and RNA from blood, microbial spores and replicates DNA and optically measures the results. The technology can reduce analysis time from around two days to just $30 \mathrm{~min}$ utes, for applications including diagnosis of 
infectious disease, cancer and the detection of biothreats such as anthrax. Its sensitivity is comparable to lab-based PCR, according to Cepheid's senior vice president of development, Bill McMillan.

\section{Automated sample preparation}

For PCR, as for other microfluidics applications, sample preparation remains a potential bottleneck. "There's this notion that samples magically get there on the chip," notes Guiseppi of ABTECH.

For PCR, extraction and preparation of DNA can take hours. Microfluidic Systems (MFSI) offers a solution with its first commercial product, BioLyser, an automated system that lyses cells, spores and viruses using ultrasonic energy, and then extracts DNA, all within disposable closed capsules. The system obviates the need for special buffers and avoids cross-contamination of samples.

The next step is to link this with PCR, and MFSI have just delivered to the US Federal Bureau of Investigation a prototype forensics analysis kit that can perform extraction

\section{BOX 1: THREE TYPES OF FLOW}

The LabChip 90 automated electrophoresis system offers scientists a one-step process for DNA and protein analysis that is faster and more quantitative than gel electrophoresis. (Courtesy of Caliper Life Sciences.)

and separation of DNA from different cell types - for example to distinguish the DNA of rapists and their victims - followed by PCR amplification, analysis and readout. If approved, it could become a conveniently portable tool for police investigators.

Meanwhile, Microchip Biotechnologies of Fremont, California, are developing a microbead capture-based platform for a next generation DNA sequencer that performs DNA library construction and amplification, fluorescent labelling and affinity capture, separation and sizing by microcapillary electrophoresis. The system is intended to reduce the cost of shotgun sequencing and resequencing by requiring only nanoliter volumes, reducing reagent consumption by 100 -fold.

The small size of microfluidic channels means that viscosity and surface tension tend to impede flow far more than for larger volumes of liquid. As a result, any of three different types of forces may be chosen to drive the flow of microfluidic devices: electro-osmosis, pressure-driven laminar flow and centrifugal force ${ }^{6}$.

To drive electro-osmosis, or the movement of charged molecules across an electrical current, Micronit's custom-designed microreactors, for example, use a high voltage to create an electrical potential across microfluidic channels. Electro-osmosis also forms the basis for BioRad's Experion automated benchtop electrophoresis system, and Agilent's 2100 Bioanalyzer and 5100 automated Lab-on-a-Chip. Caliper Life Sciences' microfluidic devices use both electrokinetic and pressure-driven flow.

Laminar flow devices, in which fluids flow smoothly and without turbulence, require pumps to deliver high pressures, up to 100 bars. Micronics, for example, offers Microflow, a software-controlled ultra-low-pulse pump system for injection and analysis of submicroliter volumes of samples and reagents in microfluidic Lab Cards. The advantage of pressure-driven flow is that it can be used to move both charged and uncharged molecules, cells and beads.

Meanwhile, the Swedish company Gyros makes use of centrifugal force in its compact disc (CD)-style solution, called Gyrolab, for customers in proteomics and the large instruments research market. Liquid is drawn into distribution channels by capillary action and then spun inside a workstation, driving precise nanoliter volumes through hundreds of individual microstructures simultaneously. The system enhances reproducibility and allows parallel processing, thus reducing reaction time, and avoids pipetting errors. CDs with customized surface chemistries have been developed for applications such as protein and cytokine quantification by immunoassay (with detection by fluorescence), and peptide concentration measurement. The CD can be cut into pieces and placed directly into certain brands of mass spectrometers for analysis, such as those supplied by Applied Biosystems Inc., Bruker and Shimatsu. Gyros is seeking partners to develop this and other applications, including in vitro diagnostics.

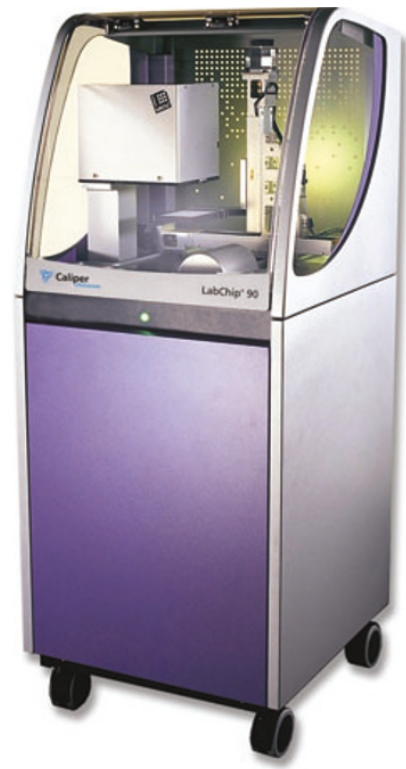

\section{Move over gel electrophoresis!}

The use of microfluidic devices based on electrophoresis has the potential to replace traditional one- and two-dimensional gel electrophoresis for the separation of biological molecules, such as proteins, RNA and DNA. Agilent Technologies' 2100 Bioanalyzer performs electrophoretic separation and quantification of DNA, RNA or proteins, all within a fingernail-sized glass chip. One application now being explored is its use in mitochondrial DNA analysis, a technique that is particularly useful in genetic typing of small or degraded samples for victim identification and forensic DNA profiling. Other applications include genomics, proteomics, drug development and manufacture, food safety and homeland security. The chip can hold and analyze up to 12 samples in just 30 minutes.

The more recent Agilent Technologies' 5100 automated Lab-on-a-Chip platform fully automates the entire process of sample preparation from standard 96- or 384-well plates, analysis and digital data readout and storage. Applications include quality control of PCR fragments prior to spotting to microarrays, target validation or sequencing, and high-throughput protein purification.

BioRad Laboratories' automated benchtop assay system Experion, also separates proteins and RNA and analyzes RNA for purity, with the advantages of being faster, allowing automation, and requiring smaller amounts of samples and reagents than traditional gel electrophoresis. 
Caliper Life Sciences has produced Lab Chip 90, an automated DNA and protein gel electrophoresis system, which produces digitized data and bypasses the need for scanning gels. And through collaboration with Agilent Technologies, its Lab Chip 3000 drug discovery system is proving popular with major pharmaceutical companies for screening organic compound libraries in enzymatic and cell-based assays. It includes proprietary 'Sipper chips' to automatically bring in samples stored in microtiter plates. "Anything done with agarose gels can be done with chips, with the advantage that results are more reproducible and data can be archived and shared with colleagues," says Andrea Chow of Caliper Life Sciences.

Caliper's collaborators include Predicant for the production of a microfluidics-based protein separation system for the analysis of complex protein patterns in blood, Affymetrix for applications in pharmacogenomics and personalized medicine, and Bio-Rad Laboratories.

\section{Drug discovery}

The pharmaceutical industry is investing substantially in microfluidics as a way to expand screening capacity at several stages in the drug discovery process.

One present bottleneck is the separation of biological compounds by liquid chromatography, for studies including drug metabolism and pharmacokinetic assays. To speed up the time to results, Nanostream

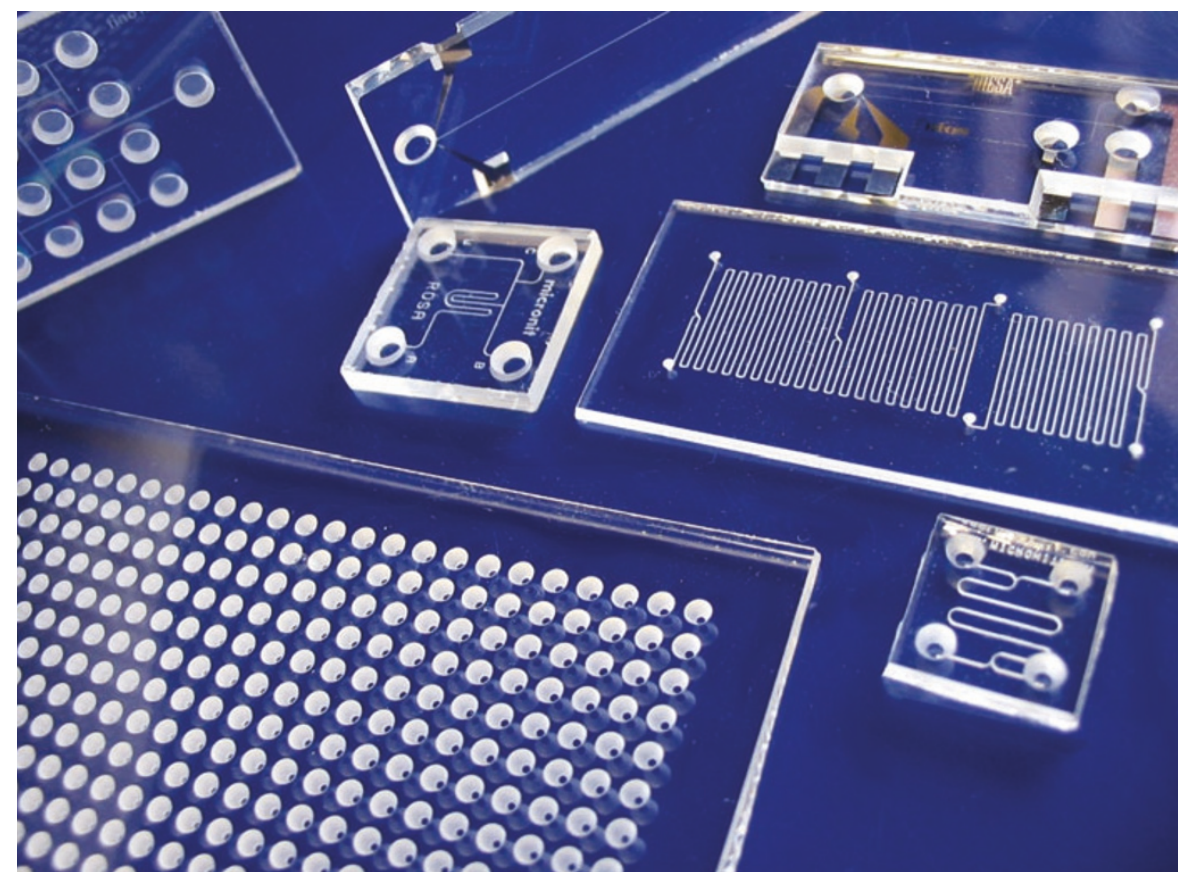

Micronit's wide range of customized glass chips can include integrated electrodes, microreactors and titer-plate format. (Courtesy of Micronit Microfluidics.)

offers the Veloce system that performs 24 simultaneous separations by micro parallel liquid chromatography, in 24-column microfluidic Brio cartridges. An add-on software-controlled fraction collector collects each eluant at fixed time intervals ready for analysis by mass spectrometry. This avoids the delay associated with serial liquid chromatography runs, and "lets the mass spectrometer go as fast as it can go", according to Nanostream's director of marketing, Surekha Vajjhala.

Agilent Technologies is applying the inject nozzle technology of its parent company, Hewlett Packard, to the same problem, providing a microfluidics-based interface between high-performance liquid chromatography (HPLC) and mass

\section{BOX 2: INSIDE A MICROCHIP}

Microchips consist of channels and chambers either etched or sand-blasted into glass or silicon, or molded into plastic polymer chips, and can incorporate valves, pumps and mixing chambers to control the flow and the mixing of reagents. Manufacturers often use proprietary technology - for example, that used by Micronit to bond together several glass layers without the need for glue- to create multiple channel depths on a single chip.

The channels of microfluidic chips vary in size and shape depending on the application, and tend to be less than 100 micrometers in diameter. For the movement of cell suspensions, for example, channels are usually at least 50 microns in diameter to minimize the risk of clogging, whereas for DNA solutions, less than 10 microns works fine, according to Ronny van't Oever of Micronit. "As engineers, we always want to go to the limits of the technology, but that might not be what the customer needs," says Thomas Strange of ThinXXS.

ThinXXS produces a 'snake mixer slide' containing undulating channels that permit fluids to be mixed intentionally. In contrast, Microfluidic ChipShop's microchips consist of glass slides with $\mathrm{H}$-shaped channels to permit two fluids to flow in opposite directions without mixing-for example, to clean up PCR solutions. Four microfluidic slides fit together into a standard 384-well microtiter plate frame for use in highthroughput robotic systems. Bio-Rad Laboratories' Experion chips consist of several cross-shaped channels filled with liquid polymer, into which up to ten samples can be driven by an electric field for size-based separation.

Channel number also varies considerably. MicroParts, for example, produce a stamp-sized Lilliput chip for microorganism identification and antibiotic resistance assays. It consists of 96 reaction cavities, each with a volume of 1.8 microliters- one hundredth of the volume of conventional microtiter plates. The cavities are connected via microchannels to a central reservoir, allowing simultaneous and automatic filling of all reaction cavities within a minute. 
spectrometry (MS). Its HPLC-Chip/MS miniaturizes HPLC to nanoscale volumes, integrating sample preparation, separation and electrospray ionization onto a single chip smaller than a credit card. The device mounts directly onto a mass spectrometer, and has the advantages of reduced sample size, and enhanced separation and sensit-i क vity compared to standard HPLC.

The need for functional cell-based assays causes a bottleneck during lead optimization and target validation. Sweden-based Cellectricon addresses this with Dynaflow, a computer controlled microfluidic chip with inbuilt patch-clamp and drugdispenser technology that speeds up drug screening against whole cell ion channels. Customers include AstraZeneca, Merck and GlaxoSmithKline.

\section{Microarrays}

One of the biggest growth markets for applying microfluidics in the life sciences is microarray technology, according to a 2003 Frost and Sullivan report, which predicted 32 percent annual growth rate in this area ${ }^{5}$.

Companies developing tools in this area include Affymetrix, with a license from Caliper Life Sciences to apply microfluidics technology to nucleic acid separation, BioRad Laboratories, Invitrogen, Perkin Elmer and the European consortium LICOM.

Invitrogen's three-dimensional microarray DNA chip, XeoChip, contains thousands of three-dimensional nanochambers in an area the size of a dime. Each chamber serves as a protected reaction vessel for DNA synthesis and subsequent bioassay for use with conventional microarray scanners. The same system can be adapted for the synthesis of RNA, peptides and other organic molecules.

Microdrop Technologies' has an inkjet technology-inspired microfluidic nozzle system that uses brief voltage-generated pressure pulses to dispense tiny drops of between 30 and 100 microliters from glass pipettes onto a target surface, at two meters per second. This is licensed exclusively to Perkin Elmer for microarray production, using eight such nozzles in parallel to be compatible with microtiter plate systems. The nozzles are made of glass to allow the use of "aggressive organic solvents and $\mathrm{pH}$," according to managing director Wilhelm Mayer.

\section{BOX 3: BUG DETECTORS}

The recent expansion of biodefense concerns has created a market for microfluidicsbased devices for the rapid detection and monitoring of infectious organisms. Together with Agilent Technologies, Invitrogen is marketing the PathAlert detection system, a PCR-based system for screening sample- and water-borne infectious agents including those responsible for anthrax, plague and tularaemia. The system combines Invitrogen's PathAlert detection kits with the Agilent 2100 Bioanalyzer or the Agilent 5100 automated Lab-on-a-chip platform, to allow higher throughput analysis, quantification and monitoring of target organisms.

One of the challenges in applying microfluidics technology to biodefense screening is how to overcome the problem of low concentration of target pathogens - analysis of just a few microliters of air may not detect their presence, for example. To this end, Microfluidic Systems Inc., in collaboration with the US Department of Homeland Security and the US Army, including USAMRIID, the lead medical research laboratory for the US Biological Defense Research Program, is creating a microfluidics-based rapid pathogen detection system that concentrates and identifies viruses, bacteria and toxins in less than five minutes. The system depends upon the flow of air or liquid across a series of deep-etched pillars, 5 microns tall and 150 microns in diameter, which together present a sufficiently large surface area - on the order of centimeters - to capture DNA, proteins or cells and elute into 50-microliter fractions.

"That's the critical step, to go from tens of milliliters down to 50 microliters," says M. Allen Northrup, president and CEO of MFSI and long-time microfluidics engineer. The same is true for trying to bring high-sensitivity diagnostics down to the scale of microfluidics: a single virus in a milliliter of blood could easily go undetected in microfluidic devices unless sample preparation involves a concentration step.

Meanwhile, Microchip Biotechnologies is using its microbead capture technology to concentrate pathogens from dilute samples of air, water and food from large volumes to milliliter and microliter volumes, before transferring the sample into a microfluidic device for processing and analysis of DNA, RNA or proteins. Cepheid's solution is to use different kinds of filters to capture cells for analysis on GeneXpert, and a solid resin for concentrating nucleic acids, allowing larger volumes of sample to be used.

Similarly, LICOM has developed a silicon chip with tiny nozzles that dispense 96 droplets of different liquids simultaneously onto a glass slide in a contact-free manner, driven by a pressure pulse.

\section{Chips galore}

Many companies in Europe and the United States provide both off-the-shelf microfluidics components, and custom design and manufacturing services, including LioniX, Micronics Inc., Boehringer-Ingelheim's MicroParts, Silicon Valley Scientific Inc., Microdrop and LICOM (Box 2).

Customers are wide ranging according to Strange of ThinXXS, who target device makers in the life sciences, pharma, healthcare, environmental monitoring and food monitoring. Among them is Californiabased Pelikan Technologies, with a blood glucose monitoring device due to be launched in the near future, and Denmarkbased Sophion Bioscience for an automated system for ion channel analysis that replaces traditional patch clamping. LICOM's collaborations include the development of microfluidic-based high-throughput screening and the manufacture of medical devices such as insulin infusion pumps, nebulizers for inhalers and other drug delivery systems.

Some producers focus on using traditional materials, glass and silicon. Micronit Microfluidics, for example, produces glass and fused silica chips for capillary electrophoresis in high-throughput systems, for anyone from "multibillion dollar international companies to research groups", according to R\&D director, Ronny van't Oever. Glass and fused silica, he says, have the benefits of being optically transparent, resistant to high temperatures and chemically inert, and are ideally suited for applications including HPLC, electrophoresis and automated high-throughput screening of drug candidates on whole cells. 
In contrast, Microfluidic ChipShop and ThinXXS produce plastic polymer-based microfluidic chips with the potential advantages of lower cost and ease of chemical modification compared to glass or silicon. These are ideally suited to use in diagnostics and bioanalysis, and offer the freedom to introduce fluidic fittings and structures for injecting or collecting samples, according to Claudia Gaertner of Microfluidic ChipShop.

\section{Making sense}

Various devices may be incorporated for the control of microfluidic flow and delivery. For example, Zurich-based Sensirion attaches electronically controlled flow sensors to the outside of microfluidic tubing for measuring pressure and flow rates down to the rate of $0.5 \mathrm{nl} / \mathrm{min}$. This permits the detection of problems such as changes in flow caused by vibrations, clogging — owing to cells or other particles-and leakage. Customers in both research institutes and industry are using these either directly or incorporating them into complex instruments, including for the development of drug delivery systems, or, as in the collaboration with Dionex Corporation, for processes such as liquid chromatography.

ABTECH Scientific, Micronit and Microdrop, also offer custom design services for the use of biosensors. ABTECH Scientific's microfabricated electrodes can be placed directly into a flowing stream to detect or capture target biomolecules. Micronit also provides integrated electrodes, for example, for the creation of bead- or cell-sorting chips, or for measuring blood contamination with metals such as lithium.

With such a plethora of microfluidic tools and devices already, or soon to arrive, on the market, only the sky seems to be the limit (Box 3). There still remains, however, the challenge of persuading scientists to fully embrace the technology. "The world is macro-there are sequencers and mass spectrometers and other big pieces of equipment. You're not going to tell them overnight that they should get rid of that!" admits Hedine of Micronics.

Micronit's van't Oever agrees that there may be some psychological barrier to switching to a microfluidic scale. "People are used to doing things in a certain way and don't like change." But, he says, "There are a lot more possibilities that people think."

Julie Clayton is a freelance writer in the UK (julie.p.clayton@blueyonder.co.uk).

1. Barry, R. \& I vanov, D. J. Nanobiotechnology 2, 2 (2004).

2. Lion, N. et al. Eectrophoresis 24, 3533-3562 (2003).

3. Mitchell, P. Nat. Biotechnol. 19, 717-721 (2001).

4. Ducre, J \& \& Zengerle, R. FlowMap: microfluidics roadmap for the life sciences (http:// www. microfluidics-roadmap.com/).

5. World Microfluidics / Lab-on-a-Chip Markets. (Frost \& Sullivan, 2003).

6. Weigl, B.H., Bardell, R.L. \& Cabrera, C.R. Adv. Drug Deliv. Rev. 55, 349-377 (2003). 


\section{SUPPLIERS GUIDE: COMPANIES OFFERING PRODUCTS AND SERVICES USING MICROFLUIDICS}

\section{Company}

ABTECH Scientific Inc.

Advalytix

Affymetrix, Inc.

Agilent Technologies

Bartels Mikrotechnik

BioMicro Systems Inc.

Bio-Rad Laboratories

Boehringer Ingelheim microParts $\mathrm{GmbH}$

Caliper Life Sciences

Cellectricon $A B$

Cepheid

Ciphergen

Eksigent Technologies, LLC

Fluidigm

Genoptix

Gyros

HandyLab

Invitrogen

LioniX

Liquid Handling Competence Centre (LICOM)

microdrop Technologies GmbH

MicroFluidic Systems

microfluidic ChipShop GmbH

Micronics, Inc.

Micronit Microfluidics bv

Nanostream

Predicant Biosciences

Sensirion AG

Shimadzu Corporation

Silicon Valley Scientific, Inc.

thinXXS

Zyomyx, Inc.
Web address

http:// www. abtechsci.com/

http:// www.advalytix.com/

http:// www. affymetrix.com/

http:// www. agilent.com

http:// www. bartels-mikrotechnik.de

http:// www.biomicro.com/

http:// www. bio-rad.com/

http:// www.boehringer-ingelheim.de/ microparts

http:// www. caliperls.com/

http:// www.cellectricon.com/

http:// www.cepheid.com

http:// www.ciphergen.com/

http:// www.eksigent.com/

http:// www.fluidigm.com

http:// www.genoptix.com

http:// www. gyros.com

http:// www.handylab.com

http:// www.invitrogen.com/

http:// www.lionixbv.nl/

http:// www.licom.net

http:// www. microdrop.de

http:// www. microfluidicsystems.com

http:// www. microfluidicchipshop.de/

http:// www. micronics.net/

http:// www. micronit.com/

http:// www. nanostream.com

http:// www. predicant.com

http:// www. sensirion.com

http:// www. shimadzu.com/

http:// www.svsci.com/

http:// www.thinxxs.com/

http:// www.zyomyx.com/ 